\title{
On some theorems of Littlewood and Selberg, IV
}

\author{
by
}

K. RAmaChandra and A. SAnkARAnARAYAnAn (Bombay)

\section{Dedicated to the eighty-first birthday of} Professor Paul Erdös

1. Introduction and notation. As usual we write $s=\sigma+i t$, and

$$
\begin{aligned}
\zeta(s) & \equiv \sum_{n=1}^{\infty}\left(n^{-s}-\int_{n}^{n+1} u^{-s} d u\right)+\frac{1}{s-1}, \quad \sigma>0, \\
& =\sum_{n=1}^{\infty} n^{-s}=\prod_{p}\left(1-p^{-s}\right)^{-1}, \quad \sigma>1 .
\end{aligned}
$$

In the last product $p$ runs over all primes $2,3,5,7,11, \ldots$ The object of this note is to prove the following theorem.

THEOREM 1. There exist effective absolute positive constants $C$ and $C^{*}$ with the following property. Let $T \geq 20, H=C \log \log \log T$ and $\zeta(s) \neq 0$ in $\left(1 / 2+(10 \log \log T)^{-1}<\sigma \leq 1, T-H \leq t \leq T+H\right)$. Then there is at least one zero of $\zeta(s)$ in the disc of radius $C^{*}(\log \log T)^{-1}$ with centre $1 / 2+i T$.

R e mark 1 . The proof of this theorem depends on Theorem 1 of our earlier paper [3], and a significant use of Ramachandra's kernel function of the third order, namely $R_{3}(z) \equiv \operatorname{Exp}(e-\operatorname{Exp}(\cos z))$. These kernels were known to Ramachandra for a long time. Ramachandra's kernel function of the second order, namely $R_{2}(z) \equiv \operatorname{Exp}\left((\sin z)^{2}\right)$, was used by him and his collaborators in various papers. Besides these the proof uses Borel-Carathéodory theorem and Hadamard's three circles theorem (the application of these last two theorems is similar to that explained in [4], pp. 210, 211).

Remark 2. A more complicated application of Borel-Carathéodory theorem and Hadamard's three circles theorem was employed by E. C. Titchmarsh to give an alternative (simpler) proof of a theorem of J. E. Littlewood (see [4], Theorem 9.12, p. 224). Littlewood's theorem asserts that given any $t>0$ there exists at least one zero $\varrho=\beta+i \gamma$ with $|t-\gamma| \leq D_{1}(\log \log \log (|t|+$ 
$\left.\left.10^{100}\right)\right)^{-1}$. Our theorem asserts that $|t-\gamma| \leq D_{2}\left(\log \log \left(|t|+10^{100}\right)\right)^{-1}$ if we assume Riemann hypothesis. Of course the result just mentioned is not difficult to prove. But Theorem 1 is not so easy to prove and is also of sufficient interest in itself.

Remark 3. It is possible to generalize Theorem 1 to functions $F(s)$ which have an Euler product and a functional equation. But the fact that there is at least one zero-free region for $F(s)$ required by Theorem 1 is not known for any such $F(s)$. Hence we have to restrict $F(s)$ to ordinary $L$-functions and $L$-functions of quadratic fields. In these cases we can prove that

$$
\frac{1}{U} \int_{U}^{2 U}|F(1 / 2+i t)|^{2} d t \leq(\log U)^{A} \quad(U \geq 3)
$$

where $A>0$ is some constant. This last condition on the mean-value ensures such a zero-free region for $F(s)$. In fact under this condition the number of zeros of $F(s)$ in $\left(\operatorname{Re} s \geq \sigma_{0},|\operatorname{Im} s| \leq U\right)$ is $\leq U^{4\left(3-2 \sigma_{0}\right)^{-1}}(\log U)^{B}$ where $B>0$ is a constant which depends on $A$.

Remark 4. While dealing with applications of Theorem 1 for $U \leq$ $T \leq 2 U$ it is convenient to prove it with $(10 \log \log U)^{-1}$ in place of $(10 \log \log T)^{-1}$. This follows from the method of our proof. Also deeper results like

$$
\frac{1}{U^{\prime}} \int_{U}^{U+U^{\prime}}|\zeta(1 / 2+i t)|^{2} d t \leq(\log U)^{A} \quad(U \geq 3)
$$

with $U^{\prime}=U^{1 / 3}$ due to R. Balasubramanian [1] and $U^{\prime}=U^{7 / 22+\varepsilon}$ due to D. R. Heath-Brown and M. N. Huxley [2] are known. These results imply the existence of intervals of the type $T \leq t \leq T+H$ contained in $\left[U, U+U^{\prime}\right]$ for which $\sigma>1 / 2+(10 \log \log U)^{-1}$ are zero free. Theorem 1 has applications to such cases also.

Remark 5. The following result may be of some interest. Let $D_{3}$ be any positive constant. Let $\zeta(s) \neq 0$ in the region $\sigma \geq 1 / 2+D_{3}(\log \log (|t|$ $+100))^{-1}$. Then given any real number $t>0$ there exists a zero $\varrho$ of $\zeta(s)$ such that

$$
|1 / 2+i t-\varrho| \leq D_{4}(\log \log (|\varrho|+100))^{-1}
$$

where $D_{4}$ is a constant which depends only on $D_{3}$. This is a hypothetical result, the hypothesis being weaker than Riemann's hypothesis. It is possible to obtain this result (by our method) by employing Ramachandra's kernel of the second order, namely $\operatorname{Exp}\left((\sin z)^{2}\right)$.

Re mark 6. In Section 2 we prove an estimate for Ramachandra's kernel of the third order, namely $R_{3}(z)$ mentioned in Remark 1 . In Section 3 we 
state a special case of Theorem 1 of our previous paper [3]. In Section 4 we complete the proof of Theorem 1 by using the Borel-Carathéodory theorem and Hadamard's three circles theorem.

Notation. We use $A, B, D_{1}, D_{2}, D_{3}, D_{4}, C, C^{*}, C_{1}, \ldots, C_{9}$ to denote effective positive numerical constants. The letter $\underline{a}$ will denote an effective large positive constant to be chosen at the end. We write $\alpha=1 / 2+$ $(10 \log \log T)^{-1}$.

2. An estimate for Ramachandra's kernel of the third order. The kernel in question is $R_{3}(z)=\operatorname{Exp}(e-\operatorname{Exp}(\cos z))$ where $z=x+i y$. We prove the following theorem.

TheOREM 2. Let $R=\left|R_{3}(z)\right|$ and $|x| \leq 2 e^{-|y|}$. Then for all $y$ we have $R \leq e^{e}$. Also if $|x| \leq 2 e^{-|y|}$ and $|y| \geq 1$, we have

$$
R \leq \operatorname{Exp}\left(e-\frac{1}{2} \operatorname{Exp}\left(\frac{1}{2} e^{|y|}-e^{-|y|}\right)\right) .
$$

In particular, if $|y|=\log \log \log T+2$ and $|x| \leq 2 e^{-2}(\log \log T)^{-1}$ we have $R \leq e^{e} T^{-(\log T) / 4}$.

Remark. Note that $2 e^{-2} \geq 1 / 5$.

Proof. We have

$$
\begin{aligned}
R & =\operatorname{Exp}\left\{e-\operatorname{Re} e^{\cosh y \cos x-i \sinh y \sin x}\right\} \\
& =\operatorname{Exp}\left\{e-e^{\cosh y \cos x} \cos (\sinh y \sin x)\right\} ;
\end{aligned}
$$

since this expression depends only on $|x|$ and $|y|$ we may suppose that $x>0$ and $y>0$. Put $\theta=(\sinh y \sin x)$ and let $x \leq 2 e^{-y}$. Then $0 \leq \theta \leq \frac{1}{2} x e^{y} \leq 1$ and so

Thus

$$
\cos \theta=1-\frac{\theta^{2}}{2 !}+\frac{\theta^{4}}{4 !}-\ldots \geq 1-\frac{\theta^{2}}{2} \geq \frac{1}{2} .
$$

Next

$$
R \leq \operatorname{Exp}\left(e-\frac{1}{2} e^{\cosh y \cos x}\right) .
$$

$$
\begin{aligned}
\cosh y \cos x & =(\cosh y)\left(1-\frac{x^{2}}{2 !}+\frac{x^{4}}{4 !}-\ldots\right) \\
& =(\cosh y)\left(1-\frac{x^{2}}{2 !}+\frac{x^{4}}{4 !}\left(1-\frac{x^{2}}{5.6}\right)+\frac{x^{8}}{8 !}\left(1-\frac{x^{2}}{9.10}\right)+\ldots\right) \\
& >(\cosh y)\left(1-\frac{x^{2}}{2}\right) \geq \frac{1}{2} e^{y}\left(1-\frac{1}{2}\left(2 e^{-y}\right)^{2}\right) \geq \frac{1}{2} e^{y}-e^{-y} .
\end{aligned}
$$

This proves the first two assertions. To prove the last assertion let $|y|=$ $\log \log \log T+2$ and $|x| \leq 2 e^{-|y|}=2 e^{-2} \log \log T$. Then by the second as- 
sertion of Theorem 2 we have

$$
\begin{aligned}
R & \leq \operatorname{Exp}\left(e-\frac{1}{2} \operatorname{Exp}\left(\frac{1}{2} e^{2} \log \log T-e^{-2}(\log \log T)^{-1}\right)\right) \\
& =\operatorname{Exp}\left(e-\frac{1}{2}(\log T)^{e^{2} / 2} \operatorname{Exp}\left(-e^{-2}(\log \log T)^{-1}\right)\right) \\
& \leq \operatorname{Exp}\left(e-\frac{1}{4}(\log T)^{e^{2} / 2}\right)=e^{e} T^{-(\log T) / 4}
\end{aligned}
$$

This proves Theorem 2 completely.

R e m a r k. To prove Theorem 1 we may assume that $T$ exceeds any large positive constant since we may increase $C^{*}$ to cover smaller values of $T$.

3. An application of our previous results to $\zeta(s)$. In this section we record a special case of Theorem 1 of [3], as Theorem 3 .

TheOREM 3. Suppose $\zeta(s) \neq 0$ in $\left(\sigma>\alpha=\frac{1}{2}+(10 \log \log T)^{-1}, T-H \leq\right.$ $t \leq T+H)$. Then for $(\sigma \geq \alpha, T-H / 2 \leq t \leq T+H / 2)$ we have

$$
|\zeta(\sigma+i t)| \leq \operatorname{Exp}\left(C_{1}(\log T)(\log \log T)^{-1}\right)
$$

and for $\left(\alpha+C_{2}(\log \log T)^{-1} \leq \sigma \leq 3 / 4, T-H / 2 \leq t \leq T+H / 2\right)$ we have

$$
|\log \zeta(\sigma+i t)| \leq C_{3}(\log T)^{2-2 \sigma}(\log \log T)^{-1} .
$$

Also for $(\sigma \geq 3 / 4, T-H / 2 \leq t \leq T+H / 2)$ we have

$$
|\log \zeta(\sigma+i t)| \leq C_{4}(\log T)^{1 / 4}(\log \log T)^{-1} .
$$

Corollary. For $(\sigma \geq 1 / 2, T-H / 4 \leq t \leq T+H / 4)$ we have

$$
|\zeta(\sigma+i t)| \leq \operatorname{Exp}\left(C_{5}(\log T)(\log \log T)^{-1}\right) .
$$

Proof of the Corollary. To get the inequality of the Corollary in $\left(1-\alpha-10(\log \log T)^{-1} \leq \sigma \leq 1-\alpha, T-H / 2 \leq t \leq T+H / 2\right)$ we can apply the first assertion of Theorem 3 and the functional equation. After this we have simply to apply the maximum modulus principle to

$$
\zeta(s+z) R_{3}(z)
$$

where $s=\sigma+i t(1-\alpha \leq \sigma \leq \alpha, T-H / 4 \leq t \leq T+H / 4)$ is any point in question and $z=x+i y$ is on the boundary of the rectangle defined by $|x| \leq(5 \log \log T)^{-1}$ and $|y|=\log \log \log T+2$. We have only to apply Theorem 2.

4. Completion of the proof. We borrow the Borel-Carathéodory theorem from page 174 of Titchmarsh's book [5].

THEOREM 4. Let $f(z)$ be an analytic function regular for $|z| \leq R$ and let $M(r)$ and $A(r)$ denote as usual the maximum of $|f(z)|$ and $R\{f(z)\}$ on $|z|=r$. Then for $0<r<R$,

$$
M(r) \leq \frac{2 r}{R-r} A(R)+\frac{R-r}{R+r}|f(0)| .
$$


Remark. We have stated the theorem in the notation of Titchmarsh's book. The letter $R$ should not be confused with our notation in Section 2 . In Theorem 4, $R\{f(z)\}$ denotes the real part of $f(z)$.

We also borrow Hadamard's three circles theorem from the same book (see [5], p. 172).

THEOREM 5. Let $f(z)$ be an analytic function regular for $r_{1} \leq|z| \leq r_{3}$. Let $r_{1}<r_{2}<r_{3}$ and let $M_{1}, M_{2}, M_{3}$ be the maximum of $|f(z)|$ on the three circles $|z|=r_{1}, r_{2}, r_{3}$ respectively. Then

$$
M_{2}^{\log \left(r_{3} / r_{1}\right)} \leq M_{1}^{\log \left(r_{3} / r_{2}\right)} M_{3}^{\log \left(r_{2} / r_{1}\right)} .
$$

From now on we write $L_{1}=\log T, L_{2}=\log \log T$, and we assume that $\zeta(s) \neq 0$ in $\left(\sigma \geq 1 / 2-10 a L_{2}^{-1}, \| t-T \mid \leq 10 a L_{2}^{-1}\right)$. We obtain a contradiction by a suitable application of Theorems 4 and 5 . We put $z_{0}=\alpha+$ $\left(C_{2}+a\right) L_{2}^{-1}+i T$.

Lemma 1. Let $f(z)=\log \zeta\left(z+z_{0}\right)$. Then with $R=6 a L_{2}^{-1}$ and $r=4 a L_{2}^{-1}$ we have

$$
M(r) \leq C_{6} a L_{1} L_{2}^{-1} .
$$

Proof. By using the asymptotic properties of the conversion factor in the functional equation for $\zeta(s)$, we see that $A(R)$ does not exceed a constant times $a L_{1} L_{2}^{-1}$. Certainly $|f(0)| \leq C_{7} e^{-a} L_{1} L_{2}^{-1}$. This completes the proof.

It is to be noted here that we have used Theorem 3 and its corollary to get the bounds for $A(R)$ and $|f(0)|$ required for the application of Theorem 4 .

Lemma 2. As before let $f(z)=\log \zeta\left(z+z_{0}\right), r_{1}=L_{2}^{-1}, r_{2}=2 a L_{2}^{-1}$ and $r_{3}=4 a L_{2}^{-1}$. We have

$$
\left(M\left(2 a L_{2}^{-1}\right)\right)^{\log (4 a)} \leq\left(C_{8} e^{-a} L_{1} L_{2}^{-1}\right)^{\log 2}\left(C_{6} a L_{1} L_{2}^{-1}\right)^{\log (2 a)} .
$$

Proof. We have $M_{2}=M\left(2 a L_{2}^{-1}\right)$ and $M_{1} \leq C_{8} e^{-a} L_{1} L_{2}^{-1}$ by Theorem 3 and $M_{3} \leq C_{6} a L_{1} L_{2}^{-1}$ by Lemma 1 . Hence the lemma follows from Theorem 5 .

Lemma 3. We have $\log \left|\zeta\left(z_{0}-2 a L_{2}^{-1}\right)\right| \geq C_{9} a L_{1} L_{2}^{-1}$ and so

$$
M\left(2 a L_{2}^{-1}\right) \geq C_{9} a L_{1} L_{2}^{-1} \text {. }
$$

Proof. The proof follows by the functional equation and the lower bound for $\log |\zeta(s)|$ in $\left(\alpha+C_{2} L_{2}^{-1} \leq \sigma \leq 3 / 4, T-H / 2 \leq t \leq T+H / 2\right)$ provided by Theorem 3 . This proves the lemma.

The proof of Theorem 1 is now complete since the inequalities asserted by Lemmas 2 and 3 contradict each other, if we choose for $\underline{a}$ a large constant.

Acknowledgements. The authors are indebted to the referee for a comment which helped them to correct many typographical errors. 


\section{References}

[1] R. Balasubramanian, An improvement of a theorem of Titchmarsh on the mean square of $|\zeta(1 / 2+i t)|$, Proc. London Math. Soc. (3) 36 (1978), 540-576.

[2] D. R. Heath-Brown and M. N. Huxley, Exponential sums with a difference, ibid. 61 (1990), 227-250.

[3] K. Ramachandra and A. Sankaranarayanan, On some theorems of Littlewood and Selberg. III, Ann. Acad. Sci. Fenn. Ser. A I Math. 16 (1991), 139-149.

[4] E. C. Titchmarsh, The Theory of the Riemann Zeta-Function, 2nd ed., revised and edited by D. R. Heath-Brown, Oxford Sci. Publ., Clarendon Press, 1986.

[5] - , The Theory of Functions, Oxford University Press, 1952.

SCHOOL OF MATHEMATICS

TATA INSTITUTE OF FUNDAMENTAL RESEARCH

HOMI BHABHA ROAD

COLABA

BOMBAY 400 005, INDIA

E-mail: KRAM@TIFRVAX.RES.IN

SANK@TIFRVAX.RES.IN

Received on 25.4.1994

and in revised form on 15.7 .1994 